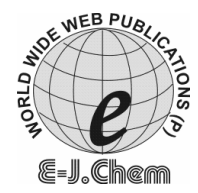

http://www.e-journals.net
ISSN: 0973-4945; CODEN ECJHAO

E-Journal of Chemistry 2009, 6(3), 747-752

\title{
Novel Synthesis and Characterization of Thiosemicarbazone Compounds Containing 4-Acyl-2-pyrazolin-5-ones
}

\author{
ARJUN K. RANA*, NAYAN R. PAREKH ${ }^{\# \text {, }}$ \\ HARISH R. DABHI ${ }^{\S}$ and SUNIL S. NADKARNI \\ ${ }^{*}$ Navajivan Science College, Dahod, \\ Gujarat University, Ahmedabad, Gujarat, India. \\ ${ }^{\#}$ Torrent Research Centre Village Bhat, \\ Gandhinagar, Ahmedabad, Gujarat, India. \\ dr.arjunsinhrana@yahoo.co.in
}

Received 9 October 2008; Revised 21 December 2008; Accepted 15 January 2009

\begin{abstract}
A novel synthesis of 4-acylthiosemicarbazone-3-methyl-1-(4methylphenyl)-2-pyrazolin-5-one by condensation of 4-acyl-3-methyl-1-(4'methylphenyl)-2-pyrazolin-5-one with thiosemicarbazide is carried out. The compounds were characterized on the basis of elemental analysis, IR, ${ }^{1} \mathrm{H}$ NMR, Mass, DSC and ${ }^{13} \mathrm{C}$ NMR spectral data. The structures were investigated for their antibacterial and antifungal activity. They are very essential to study on cerebral infarction (Free radical scavenger). A single crystal X-ray study of this thiosemicarbazones and their metal complexes is in progress.
\end{abstract}

Keywords: 4-Acyl-3-methyl-1-(4-methylphenyl)-2-pyrazolin-5-one, Thiosemicarbazone and Amine substitution, Biological activity.

\section{Introduction}

Thiosemicarbazide containing 4-acyl-2-pyrazolin-5-one derivatives form an important class of organic compounds due to their structural chemistry and biological activities, such as antibacterial, antivirus activities and cerebral infarction (Free radical scavenger) ${ }^{1-4}$. Especially heterocyclic thisemicarbazones have been the subject of extensive investigation because of their use for the biological applications is very wide ${ }^{5-7}$ as compare to those with the heteroaromatic ring containing substitution as 2 or 4 position, the thisemicarbazones have less attention ${ }^{8-10}$. Thiosemicarbazone compounds can be converted in to complexes by reaction with metal ions and the reaction product has very important uses ${ }^{2,5}$. There are different substituted amide bonds (-CONH-) in structure of these compounds, therefore most 
of them have good biological activities and there are some reports about their use as herbicides and bactericides ${ }^{11}$. In order to exploit new type of chetale extracting and biological active compound, four different kind of 4-acyl-3-methyl-1-(4'-methylphenyl)-2-pyrazolin-5-one are used in present study to react with thiosemicarbazide and new compound of 4-acylthiosemicarbazone-3methyl-1-(4'-methylphenyl)-2-pyrazolin-5-one are synthesized. Literature survey revels that few 4-acylthisemicarbazones are reported ${ }^{10-13}$ but 4-propionylthiosemicarbazone-3-methyl-1(4-methylphenyl)-2-pyrazolin-5-one (1a), 4-butyrylthiosemicarbazone-3-methyl-1-(4'-methyl phenyl)-2-pyrazolin-5-one (1b), 4-acetylthiosemicarbazone-3-methyl-1-(4`-methylphenyl)2-pyrazolin-5-one (1c) and 4-benzoylthiosemicarbazone-3-methyl-1-(4-methylphenyl)-2pyrazolin-5-one (1d) which have never been reported.

Here, we report synthesis and structural characterization of $\mathbf{1 a}, \mathbf{1 b}, \mathbf{1 c}$, and $\mathbf{1 d}$ in continuation of our earlier work ${ }^{12-17}$ on Oximes, $N$-methylthiosemicarbazones, $N$-phenyl thiosemicarbazones, semicarbazones, hydrazones, phenylhydrazones and Schiff bases with mono, di, tri-amines of 4-acyl-2-pyrazolin-5-ones. Preparation of amine substituted of thiosemicarbazones of 4-acyl-3-methyl-(4-methylphenyl)-2-pyrazolin-5-ones work is in progress, we have also prepared the single crystal of some 4-acylthiosemicarbazone-3-methyl1-(4`-methylphenyl)-2-pyrazolin-5-one compounds $\mathbf{1 a}, \mathbf{1 b}, \mathbf{1 c}$ and $\mathbf{1 d}$ and their metal complexes. Several 4-acyl-3-methyl-1-phenyl-2-pyrazolin-5-ones were prepared and applied for the separation of the metals ${ }^{18}$.

\section{Experimental}

3-Methyl-1-(4-methylphenyl)-2-pyrazolin-5-one, acetyl chloride, propionyl chloride, benzoyl chloride, butryl chloride, calcium hydroxide, acetic acid, 4-methylphenyl hydrazine, methanol, conc. hydrochloric acid, thiosemicarbazide and dioxane used were of analytical grade.

The elemental analysis C, H, N and S were determined at Gujarat Lab Ahmedabad. The IR spectra of the compounds were recorded on perkin-Elmer 2000 FTIR spectrophotometer, as $\mathrm{KBr}$ discs in the range $4000-400 \mathrm{~cm}^{-1}$ The DSC, NMR,IR, Mass and X-RPD were determine at SCICART, vallabhvidhyanagar, Gujarat (India).

\section{Synthesis}

\section{Preparation of 4-acyl-3-methyl-1-(4`-methylphenyl)-2-pyrazolin-5-one}

The reaction of 3-methyl-1-(4-methylphenyl)-2-pyrazolin-5-one with acetyl chloride, benzoylchloride, butrylchloride and propionylchloride were carried out as per earlier publication ${ }^{18}$ for the preparation of 4-acyl-3-methyl-(4-methylphenyl)-2-pyrazolin-5-ones as shown in Scheme 1.
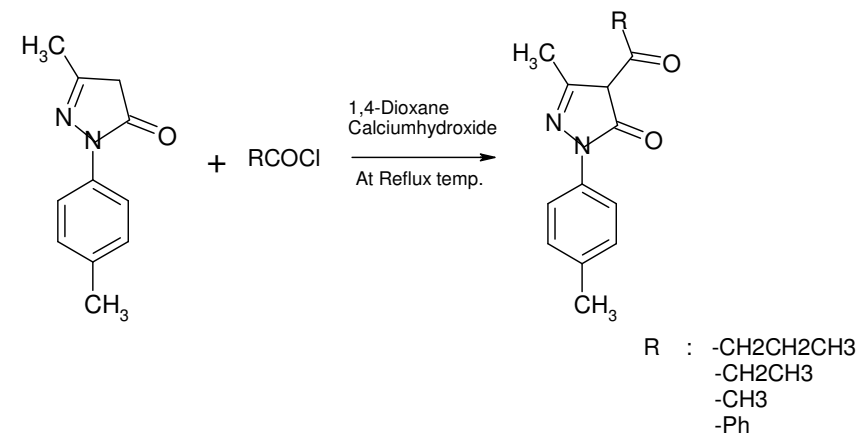

\section{Scheme 1}


1,4-Dioxane solution (250 mL) of 3-methyl-1-(4-methylphenyl)-2-pyrazolin-5-one $(25.0 \mathrm{~g})$ and propionyl chloride $(13.25 \mathrm{~mL})$ was refluxed for $2 \mathrm{~h}$ in oil bath with calcium hydroxide $(13.97 \mathrm{~g})$ and cooled to room temperature. The resulting reaction mass is added to the dilute hydrochloric acid $(45 \mathrm{~mL}$ conc. $\mathrm{HCl}$ in $200 \mathrm{~mL}$ water), the crude product was collected by filtration and washed several time with water and then dried in air. The 4-propionyl-3-methyl-1-(4-methylphenyl)-2-pyrazolin-5-one is light yellow powder and its yield is $89 \%$, Anal. Calcd. (\%) $\mathrm{C}_{14} \mathrm{H}_{16} \mathrm{~N}_{2} \mathrm{O}_{2}$; M.W: $244.29, \mathrm{C}(68.83 \%), \mathrm{H}(6.60 \%), \mathrm{N}(11.47 \%)$, found $(\%) \mathrm{C}(68.71 \%), \mathrm{H}(6.656 \%), \mathrm{N}(11.48 \%)$ for DSC (onset:99.47 ${ }^{\circ} \mathrm{C}$ and peak:101.19 ${ }^{\circ} \mathrm{C}$.) ${ }^{1} \mathrm{H}$ NMR (DMSO-d 6 ) $\delta: 1.031-2.33\left(\mathrm{t}, 5 \mathrm{H}, \mathrm{CH}_{2} \mathrm{CH}_{3}\right), 2.41\left(\mathrm{~S}, 3 \mathrm{H}, \mathrm{PZ}-\mathrm{CH}_{3}\right), 2.81\left(\mathrm{~S}, 2 \mathrm{H}, \mathrm{NH}_{2}\right.$ ), $7.2 \sim 7.5(\mathrm{~m}, 5 \mathrm{H}, \mathrm{Ph}) \mathrm{IR}(\mathrm{KBr}) \mathrm{v}: 3432(\mathrm{w})(\mathrm{N}-\mathrm{H}), 3292(\mathrm{w})(\mathrm{O}-\mathrm{H}), 1621(\mathrm{~s}) \quad(\mathrm{Pz}-\mathrm{C}=\mathrm{O}), 1553(\mathrm{~s})$, 1512(s), 1441(s)Ph, 1404(m), 1364(m)(Pz) and MASS $245 \mathrm{M}^{+}$.

4-Butyryl-3-methyl-1-(4-methylphenyl)-2-pyrazolin-5-one is light yellow powder and its yield is $85 \%$, Anal. Calcd. (\%) $\mathrm{C}_{15} \mathrm{H}_{18} \mathrm{~N}_{2} \mathrm{O}_{2}$ M.W: 258.31, C(69.74\%), $\mathrm{H}(7.02 \%), \mathrm{N}(10.84 \%)$ found $(\%) \mathrm{C}(69.56 \%), \mathrm{H}(7.12 \%), \mathrm{N}(10.85 \%)$ for DSC (onset : $82.88^{\circ} \mathrm{C}$ and peak:84.43 ${ }^{\circ} \mathrm{C}$ ) ${ }^{1} \mathrm{H}$ NMR (DMSO-d $\left.{ }_{6}\right) \quad 8: 0.9-2.33\left(\mathrm{~m}, 7 \mathrm{H}, \mathrm{CH}_{2} \mathrm{CH}_{2} \mathrm{CH}_{3}\right), 2.40\left(\mathrm{~S}, 3 \mathrm{H}, \mathrm{PZ}-\mathrm{CH}_{3}\right), 2.81(\mathrm{~S}, 2 \mathrm{H}$, $\left.\mathrm{NH}_{2}\right), 7.2 \sim 7.5(\mathrm{~m}, 5 \mathrm{H}, \mathrm{Ph}) \mathrm{IR}(\mathrm{KBr}) \mathrm{v}: 3209(\mathrm{br})\left(\mathrm{NH}_{2}\right), 3041(\mathrm{~s})(\mathrm{N}-\mathrm{H}), 1624(\mathrm{~s})(\mathrm{Pz}-\mathrm{C}=\mathrm{O}), 1541(\mathrm{~m})$, 1511(m), 1430(s) Ph, 1409(m),1382-1340(s)(Pz) and MASS $259 \mathrm{M}^{+}$.

4-Acetyl-3-methyl-1-(4-methylphenyl)-2-pyrazolin-5-one is light yellow powder and its yield is $82 \%$, Anal. Calcd. (\%) $\mathrm{C}_{13} \mathrm{H}_{14} \mathrm{~N}_{2} \mathrm{O}_{2}$ M.W: 230.26, C(67.81\%), $\mathrm{H}(6.13 \%), \mathrm{N}$ $(12.17 \%)$ found (\%) $\mathrm{C}(67.67 \%), \mathrm{H}(6.151 \%), \mathrm{N}(12.15 \%)$ for DSC (onset:101.86 ${ }^{\circ} \mathrm{C}$ and peak:103.14 ${ }^{\circ} \mathrm{C}$.) ${ }^{1} \mathrm{H}$ NMR $\left(\mathrm{DMSO}_{6}\right) \delta: 2.33\left(\mathrm{~s}, 3 \mathrm{H}, \mathrm{CH}_{3}\right), 2.40\left(\mathrm{~S}, 3 \mathrm{H}, \mathrm{PZ}_{6}-\mathrm{CH}_{3}\right), 2.39(\mathrm{~S}$, $\left.2 \mathrm{H}, \mathrm{NH}_{2}\right), 7.2 \sim 7.5(\mathrm{~m}, 5 \mathrm{H}, \mathrm{Ph}) \mathrm{IR}(\mathrm{KBr}) \mathrm{v}: 2924(\mathrm{w})(\mathrm{N}-\mathrm{H}), 2967(\mathrm{w})(\mathrm{O}-\mathrm{H}), 1635(\mathrm{~s})(\mathrm{Pz}-\mathrm{C}=\mathrm{O})$, 1552(s), 1510(s), 1441(s)Ph, 1364(m)1369(m)(Pz) and MASS $230.8 \mathrm{M}^{+}$.

4-Benzoyl-3-methyl-1-(4-methylphenyl)-2-pyrazolin-5-one is light yellow powder and its yield is $90 \%$, Anal. Calcd. (\%) $\mathrm{C}_{18} \mathrm{H}_{16} \mathrm{~N}_{2} \mathrm{O}_{2}$ M.W: 292.33, C(73.95\%), $\mathrm{H}(5.52 \%), \mathrm{N}$ $(9.58 \%)$, found (\%) $\mathrm{C}(73.37 \%), \mathrm{H}(5.52 \%), \mathrm{N}(9.55 \%)$ for DSC (onset:103.58 ${ }^{\circ} \mathrm{C}$ and peak:107.15 $\left.{ }^{\circ} \mathrm{C}.\right){ }^{1} \mathrm{H}$ NMR $\left(\mathrm{DMSO}_{-} \mathrm{d}_{6}\right) \delta: 2.22\left(\mathrm{~s}, 3 \mathrm{H}, \mathrm{Ph}-\mathrm{CH}_{3}\right), 2.33\left(\mathrm{~s}, 3 \mathrm{H}, \mathrm{PZ}-\mathrm{CH}_{3}\right), 3.5(\mathrm{~S}$, $\left.2 \mathrm{H}, \quad \mathrm{NH}_{2}\right), \quad 7.2 \sim 7.5(\mathrm{~m}, \quad 8 \mathrm{H}, \mathrm{Ph}) \quad \mathrm{IR}(\mathrm{KBr}) \quad \mathrm{v}: 3373(\mathrm{w})(\mathrm{N}-\mathrm{H}), \quad 1601(\mathrm{~s})(\mathrm{Pz}-\mathrm{C}=\mathrm{O}), 1542(\mathrm{~s})$, 1449(s)Ph, 1429(m),1387(m)(Pz) and MASS 293.19 $\mathrm{M}^{+}$.

\section{Preparation of 4-acylthiosemicarbazone-3-methyl-1-(4`methylphenyl)-2-pyrazolin-5-one}

The reaction of 4-acyl-2-pyrazoline-5-one with thiosemicarbazide produce Schiff base was reported earlier ${ }^{19-22}$. In our novel process 4-acyl-3-methyl-1--(4`-methylphenyl)-2-pyrazolin-5one react with thiosemicarbazide was carried out in ethanol or methanol at reflux temperature in water bath to get $\mathbf{1 a}, \mathbf{1 b}, \mathbf{1 c}$ and $\mathbf{1 d}$ is shown in scheme 2. All the synthesized compounds 1a, 1b, 1c and $1 \mathbf{d}$ are crystallized in aqueous ethanol as a yellow prismatic crystal.

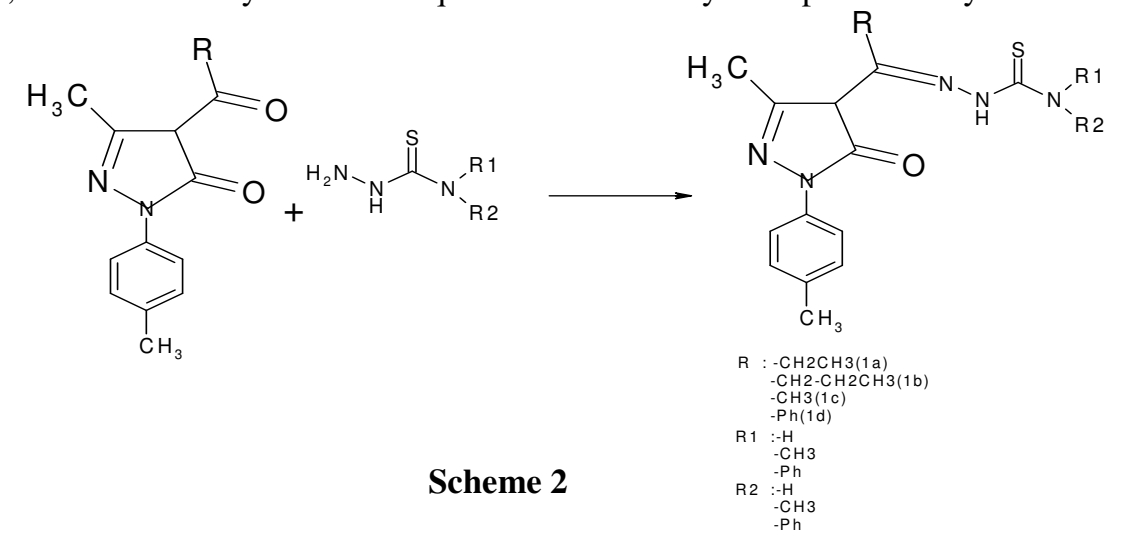


Ethanol or methanol solution $(250 \mathrm{~mL})$ of 4-propionyl-3-methyl-1-(4) methylphenyl)-2-pyrazolin-5-one $(25.0 \mathrm{~g}, 1$ mole) and thiosemicarbazide (1.1 mole) to this reaction mass added catalytic amount of acetic acid $(\sim 15-20 \mathrm{~mL})$ was refluxed for 3 to $5 \mathrm{~h}$. Cooled to room temperature. Solid light yellow to brown product filtered and washed with methanol or ethanol. 4-Propionylthiosemicarbazone-3-methyl-1-(4)methylphenyl)-2-pyrazoline-5-one (1a) is light yellow powder and its yield is $80 \%$, Anal. Calcd. (\%) $\mathrm{C}_{15} \mathrm{H}_{19} \mathrm{~N}_{5} \mathrm{OS}$ M.W: $317.4 \quad \mathrm{C}(56.76 \%), \mathrm{H}(6.03 \%), \mathrm{N}(22.06 \%)$, $\mathrm{S}(10.10 \%)$, found $(\%) \mathrm{C}(56.13 \%), \mathrm{H}(6.04 \%), \mathrm{N}(22.65 \%), \mathrm{S}(11.24 \%)$, for DSC (onset:173.82 ${ }^{\circ} \mathrm{C}$ and peak:178.88 ${ }^{\circ} \mathrm{C}$.) ${ }^{1} \mathrm{H}$ NMR (DMSO-d $\left.{ }_{6}\right) \quad \delta: 1.31-2.2(\mathrm{~m}, 5 \mathrm{H}$, $\left.\mathrm{CH}_{2} \mathrm{CH}_{3}\right), 2.33\left(\mathrm{~S}, 3 \mathrm{H}, \mathrm{PZ}-\mathrm{CH}_{3}\right), 2.80\left(\mathrm{~S}, 2 \mathrm{H}, \mathrm{NH}_{2}\right), 7.2 \sim 7.8(\mathrm{~m}, 5 \mathrm{H}, \mathrm{Ph}), 10.07(\mathrm{~S}, 1 \mathrm{H}$, NH-tsc), 12.10(S, 1H, Pz-NH) IR(KBr) v:3426(w)(N-H), 3111..2867(br,m), 1627(s)(Pz$\mathrm{C}=\mathrm{O}), 1540(\mathrm{~s}), 1510(\mathrm{~s}), 1459(\mathrm{~s})(\mathrm{Ph}), 1387(\mathrm{~m})(\mathrm{Pz}), 1364(\mathrm{~s}), 1243(\mathrm{~s}), 822(\mathrm{~m})(\mathrm{C}=\mathrm{S})$ and MASS $316 \mathrm{M}^{-13} \mathrm{C}$ NMR (DMSO-d $\left.)_{6}\right) \quad \delta: \quad 164.66(\mathrm{C}=\mathrm{S})$ 146.52(Pz-C=O) 129.03$118.54(\mathrm{Ph}) 117.94-136.42(\mathrm{C}-\mathrm{N}) 18.45\left(\mathrm{pz}-\mathrm{CH}_{3}\right)$ 14.08(C- $\left.\mathrm{CH}_{3}\right) 38.76-40.01\left(\mathrm{Ph}-\mathrm{CH}_{3}\right)$.

4-Butrylthiosemicarbazone-3-methyl-1-(4-methylphenyl)-2-pyrazolin-5-one (2b) is light yellow powder and its yield is $84 \%$, Anal. Calcd. (\%) $\mathrm{C}_{16} \mathrm{H}_{21} \mathrm{~N}_{5} \mathrm{OS}$ M.W: 331.43 $\mathrm{C}(57.98 \%), \mathrm{H}(6.39 \%), \mathrm{N}(21.13 \%), \mathrm{S}(9.67 \%)$ found $(\%) \mathrm{C}(53.03 \%), \mathrm{H}(6.06 \%)$, $\mathrm{N}(17.917 \%), \mathrm{S}(9.28 \%)$ for DSC (onset: $156.41{ }^{\circ} \mathrm{C}$ and peak:159.79 $\left.{ }^{\circ} \mathrm{C}.\right){ }^{1} \mathrm{H}$ NMR (DMSO-d $) \quad \delta: 1.031-2.33\left(\mathrm{~m}, 9 \mathrm{H}, \mathrm{CH}_{2} \mathrm{CH}_{2} \mathrm{CH}_{3}\right), 2.5\left(\mathrm{~S}, 3 \mathrm{H}, \mathrm{PZ}-\mathrm{CH}_{3}\right), 2.6\left(\mathrm{~S}, 2 \mathrm{H}, \mathrm{NH}_{2}\right)$, 7.2 7.5(m, 5H,Ph), 10.05(S, 1H, NH-tsc), 12.15(S, 1H, Pz-NH) IR(KBr) v:3742(w)(N$\mathrm{H}), \quad 3128 . .2818(\mathrm{br}, \mathrm{m}), 1634(\mathrm{~s})(\mathrm{Pz}-\mathrm{C}=\mathrm{O}), 1536(\mathrm{~s}), 1511(\mathrm{~s}), 1451(\mathrm{~s}) \mathrm{Ph}, 1372(\mathrm{~m})(\mathrm{Pz})$, 1344(s), 1229(s), 828(m) $(\mathrm{C}=\mathrm{S})$ and MASS $330 \mathrm{M}^{-13} \mathrm{C}$ NMR (DMSO-d $\left.{ }_{6}\right) \delta$ : 164.02(C=S) $146.57(\mathrm{Pz}-\mathrm{C}=\mathrm{O}) \quad 129.11-118.02(\mathrm{Ph}) \quad 118.02-136.48(\mathrm{C}-\mathrm{N}) \quad 16.29\left(\mathrm{pz}-\mathrm{CH}_{3}\right)$ 12.32(C- $\left.\mathrm{CH}_{3}\right) 39.85\left(\mathrm{Ph}-\mathrm{CH}_{3}\right)$.

4-Acetylthiosemicarbazone3-methyl-1-(4`-methylphenyl)-2-pyrazolin-5-one (1c) is light yellow powder and its yield is 90\%, Anal. Calcd. (\%) $\mathrm{C}_{14} \mathrm{H}_{17} \mathrm{~N}_{5} \mathrm{OS}$ M.W: $303.38 \mathrm{C}(55.42 \%), \mathrm{H}(5.65 \%), \mathrm{N}(23.08 \%), \mathrm{S}(10.57 \%)$; found $(\%) \mathrm{C}(55.43 \%)$, $\mathrm{H}(5.63 \%), \mathrm{N}(23.04 \%), \mathrm{S}(10.98 \%)$ for DSC (onset:203.23 ${ }^{\circ} \mathrm{C}$ and peak:205.28 ${ }^{\circ} \mathrm{C}$.) ${ }^{1} \mathrm{H}$ NMR (DMSO-d $) \quad \delta: 2.29\left(\mathrm{~s}, 3 \mathrm{H}, \mathrm{CH}_{3}\right), 2.5\left(\mathrm{~S}, 3 \mathrm{H}, \mathrm{Pz}-\mathrm{CH}_{3}\right), 2.6\left(\mathrm{~S}, 2 \mathrm{H}, \mathrm{NH}_{2}\right)$, $7.18 \sim 7.85(\mathrm{~m}, \quad 5 \mathrm{H}, \mathrm{Ph}), \quad 10.03(\mathrm{~S}, \quad 1 \mathrm{H}, \quad \mathrm{NH}-\mathrm{tsc}), \quad 12.10(\mathrm{~S}, \quad 1 \mathrm{H}, \quad \mathrm{Pz}-\mathrm{NH}) \quad \mathrm{IR}(\mathrm{KBr})$ $\mathrm{v}: 3747(\mathrm{w})(\mathrm{N}-\mathrm{H}), \quad 3214.2865(\mathrm{br}, \mathrm{m}), 1624(\mathrm{~s})(\mathrm{Pz}-\mathrm{C}=\mathrm{O}), 1540(\mathrm{~s}), 1511(\mathrm{~s}), 1472(\mathrm{~s}) \mathrm{Ph}$, 1364(m)(Pz), 1324(s), 1208(s), 827(m) $(\mathrm{C}=\mathrm{S})$ and MASS $304 \mathrm{M}+{ }^{13} \mathrm{C}$ NMR (DMSO$\left.\mathrm{d}_{6}\right) \quad \delta: 164.82(\mathrm{C}=\mathrm{S}) \quad 147.73(\mathrm{Pz}-\mathrm{C}=\mathrm{O}) \quad 129.61-118(\mathrm{Ph}) \quad 118.51-137.05(\mathrm{C}-\mathrm{N}) \quad 17.31(\mathrm{pz}-$ $\left.\mathrm{CH}_{3}\right)$ 14.82(C- $\left.\mathrm{CH}_{3}\right) 39.32-40.57\left(\mathrm{Ph}-\mathrm{CH}_{3}\right)$.

4-Benzoyl-thiosemicarbazone-3-methyl-1-(4`-methylphenyl)-2-pyrazolin-5-one(2d) is light yellow powder and its yield is $86 \%$, Anal. Calcd. (\%) $\mathrm{C}_{19} \mathrm{H}_{19} \mathrm{~N}_{5} \mathrm{OS}$ M.W: 365.45 $\mathrm{C}(62.44 \%), \mathrm{H}(5.24 \%), \mathrm{N}(19.16 \%), \mathrm{S}(8.77 \%)$; found (\%) $\mathrm{C}(56.65 \%), \mathrm{H}(5.59 \%)$, $\mathrm{N}(16.304 \%), \mathrm{S}(9.8 \%)$ for DSC (onset: $148{ }^{\circ} \mathrm{C}$ and $208.28{ }^{\circ} \mathrm{C}$; peak: $150.54{ }^{\circ} \mathrm{C}$ and $212.12{ }^{\circ} \mathrm{C}$.) ${ }^{1} \mathrm{H}$ NMR (DMSO-d 6 ) $\delta: 7.31(\mathrm{~m}, 4 \mathrm{H}, \mathrm{Ph}) ,2.51\left(\mathrm{~S}, 3 \mathrm{H}, \mathrm{PZ}-\mathrm{CH}_{3}\right), 3.31(\mathrm{~S}$, $\left.2 \mathrm{H}, \mathrm{NH}_{2}\right), 7.2 \sim 7.5(\mathrm{~m}, 5 \mathrm{H}, \mathrm{Ph}), 8.24(\mathrm{~S}, 1 \mathrm{H}, \mathrm{NH}-\mathrm{tsc}), \operatorname{IR}(\mathrm{KBr}) \mathrm{v}: 3648(\mathrm{w})(\mathrm{N}-\mathrm{H})$, 3169..2803(br,m), 1611(s)(Pz-C=O),1540(s), 1506(s)Ph, 1398(m)(Pz), 1349(s), 1242(s), 837(m) $(\mathrm{C}=\mathrm{S})$ and MASS $366 \mathrm{M}^{+13} \mathrm{C}$ NMR $\left(\mathrm{DMSO}_{-} \mathrm{d}_{6}\right) \delta: 165.48(\mathrm{C}=\mathrm{S})$ $147.23(\mathrm{Pz}-\mathrm{C}=\mathrm{O}) \quad 129.8-118(\mathrm{Ph}) \quad 118.7-141.10(\mathrm{C}-\mathrm{N}) \quad 16.31\left(\mathrm{pz}^{\left.-\mathrm{CH}_{3}\right)} \quad 14.17\left(\mathrm{C}-\mathrm{CH}_{3}\right)\right.$ 39.31-39.67( $\left.\mathrm{Ph}-\mathrm{CH}_{3}\right)$.

Above all, ligands are in crystalline form as per X-RPD patern done on X'pert PRO Panalytical using $\mathrm{Cu}$, Generator setting-45kV/40 m A; Goniometer (Pw3050/60( $\theta / \theta$ ), $240 \mathrm{~mm}$ Gonoimeter radius. 


\section{Results and Discussion}

The New Schiff base the 4-acylthiosemicarbazone-3-methyl-1-(4-methylphenyl)-2pyrazolin-5-one would be good precursors for metal complexes like Lanthanide series and others metals like $\mathrm{Co}, \mathrm{Cr}, \mathrm{Mn}, \mathrm{Mg}, \mathrm{Pd}, \mathrm{Ni}, \mathrm{Fe}, \mathrm{Zn}, \mathrm{Ca}, \mathrm{Hg}$, and $\mathrm{As}$ in bidentate or tridentate respectively.

We disclosed analytical data like elemental analysis, ${ }^{1} \mathrm{H}$ NMR, ${ }^{13} \mathrm{C}$ NMR, IR, Mass spectra, DSC and X-ray powder diffraction for new Schiff Base ligands formation confirmation, more work is going on for preparation of metal complexes of the above amine substituted thiosemicarbazones with metal complexes and single crystal structure thereof. Use of these ligands system now covers a full of areas ranging from general consideration of metal-sulphur bonding and electron delocalization in transition metal complexes to potential biological activity work is under analysis. Discussion here is restricted only to $\mathrm{O}, \mathrm{S}$ and $\mathrm{N}$ Containing ligands, especially thiosemicarbazide

\section{Conclusion}

The New Schiff base the 4-acylthiosemicarbazone-3-methyl-1-(4-methylphenyl)-2pyrazoline-5-one would be good precursors for metal complexes like Lanthanide series and others metals, syntheses were conformed by elemental analysis, ${ }^{1} \mathrm{H}$ NMR, ${ }^{13} \mathrm{C}$ NMR, IR, Mass spectra, DSC and IR.

\section{Acknowledgement}

We are grateful to Dahod Anaj Mahajam Sarvajanic Education Society and Principal, Navajivan Science College, And Dahod for the Laboratory facilities.

\section{References}

1. Lestk Vladzimirska O, Horishny V, Nektegayvew I and Pshyk I, Sci Pharm., 1997, 65, 101.

2. Rehse K and Muller U, Arch Pharm.,_(Weinheim), 1995, 328, 765.

3. Yukihito Higashi, Daisuke Jitsuikia, Kazuaki Chayamab and Masao, Recent Patents on Cardiovascular Drug Discovery, 2006, 1, 85-93.

4. Kuznetsov M I, Dement ev A I and Zhornik V V, J Mol Struct (THEOCHEM), 2001, 571, 45.

5. Dimmock J R, Vashishtha S C and Stables J P, J Med Chem., 2000, 35, 241-248.

6. Klimova T, Klimova E I, Mertinez Garcia M, Mendez stivalet J M and Ramirez L R, J Organomet Chem., 2001, 633, 137-142.

7. Bharti N, Husain K, Gonzalez Garza M T, Cyuz-Vega DE and Castro-Garza J, Bioorg Med Chem Lett., 2002, 12, 3475-3478.

8. Baldini M, Belicchi-Ferrari M, Bisceglie F, Pelosi G, Pinelli S and Trasconi P, Inorg Chem., 2003, 42, 2049-2055.

9. Santos I G, Abram U, Alberto R, Lopez E V and Sanchez A, Inorg Chem., 2004, 43, 1834-1836.

10. Liu Guang-fei, Liu lang, Hu Xin, Jia Dian-Ze and Yu Kai-Bei, Chinese J Srtuct Chem., 2006, 25(10), 1233-1237.

11. Guo D S, Huang R O and Gao R H Z G, J Chin Univ., 1996, 17(2), 255.

12. Rana A K and Shah J R, J Indian Chem Soc., 1981, 58, 1100.

13. Rana A K and Shah J R, Indian J Chem., 1982, 21A, 177.

14. Kharodawala M J and Rana A K, Synth React Inorg Met Org Chem., 2003, 33(8), 1483-1504. 
15. Rana A K, Dabhi H R and Pancholi A.M, Orient J Chem., 1996, 12, 287.

16. Rana A.K and Kharodawala M.J, Asian J Chem., 2002, 14(2), 703.

17. Rana A. K, Bhoi D.K and Dabhi H.R, Asian J Chem.,2009, 21(2).

18. Subrata Kumar Dey, Bappaditya Bag, Dilip Kumar Dey, Gramlich V, Yadong Li and Samiran Mitra, Zeitschrift für Naturforschung B., 2003, 58b, 1009-1015.

19. Jensen B S, Acta Chem Scand., 1959, 13, 1668-1670.

20. Liu Guang-fei, Liu lang, Hu Xin, Jia Dian-Ze and Yu Kai-Bei, Acta Chemica Sinica., 2004, 62(7), 697-702.

21. Tang X C, Jia D Z, Liang K, Zhang X and Zhou Z Y, J Photochem Photobiol A Chem., 2000, 134, 23.

22. Liu L and Jia D Z, J Photochem Photobiol A Chem., 2002, 153. 


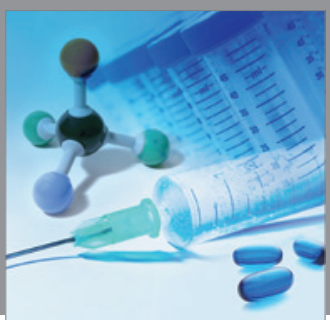

International Journal of

Medicinal Chemistry

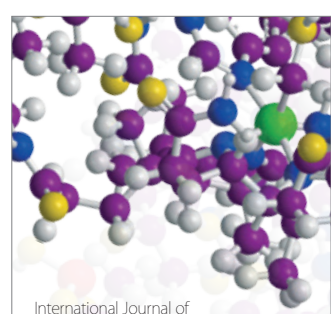

Carbohydrate Chemistry

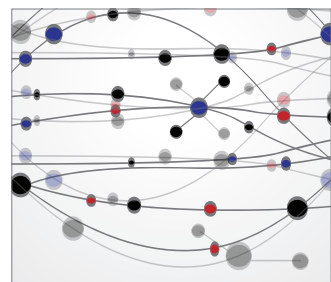

The Scientific World Journal
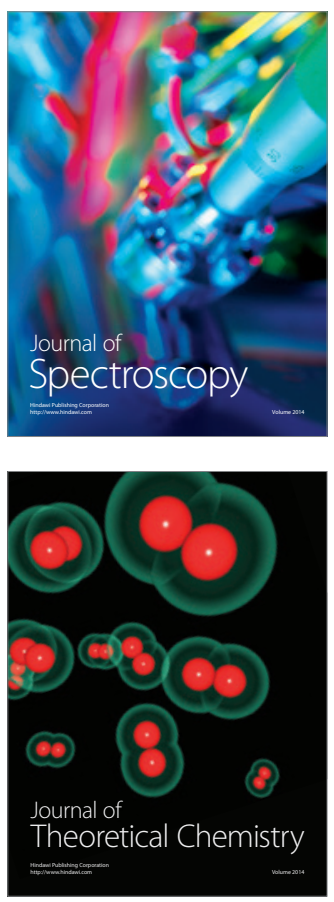
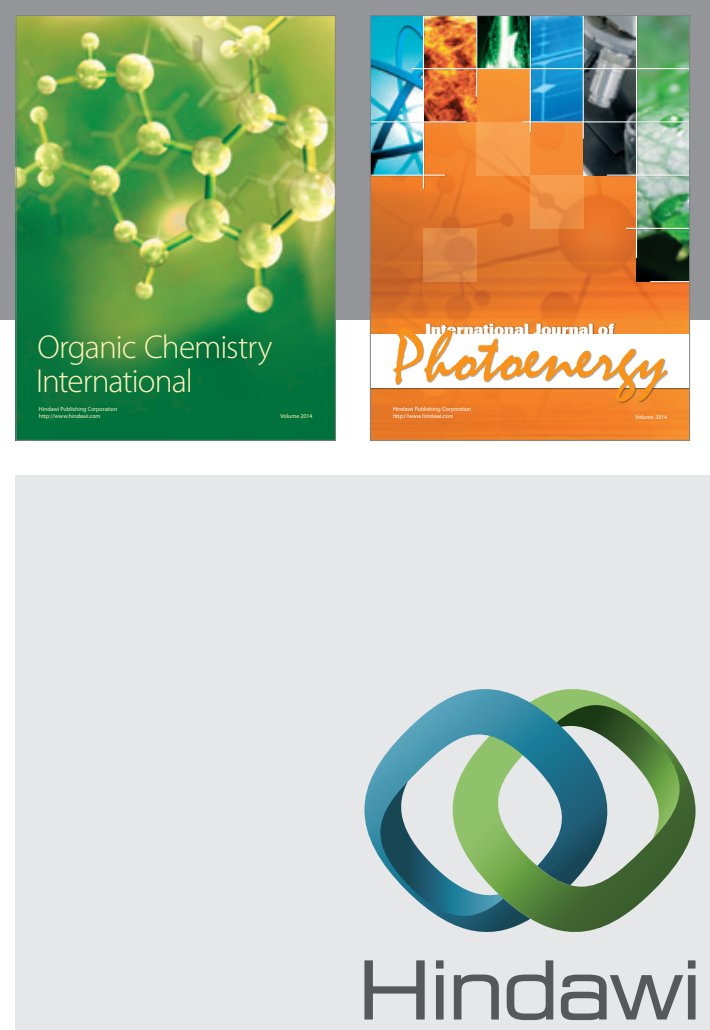

Submit your manuscripts at

http://www.hindawi.com
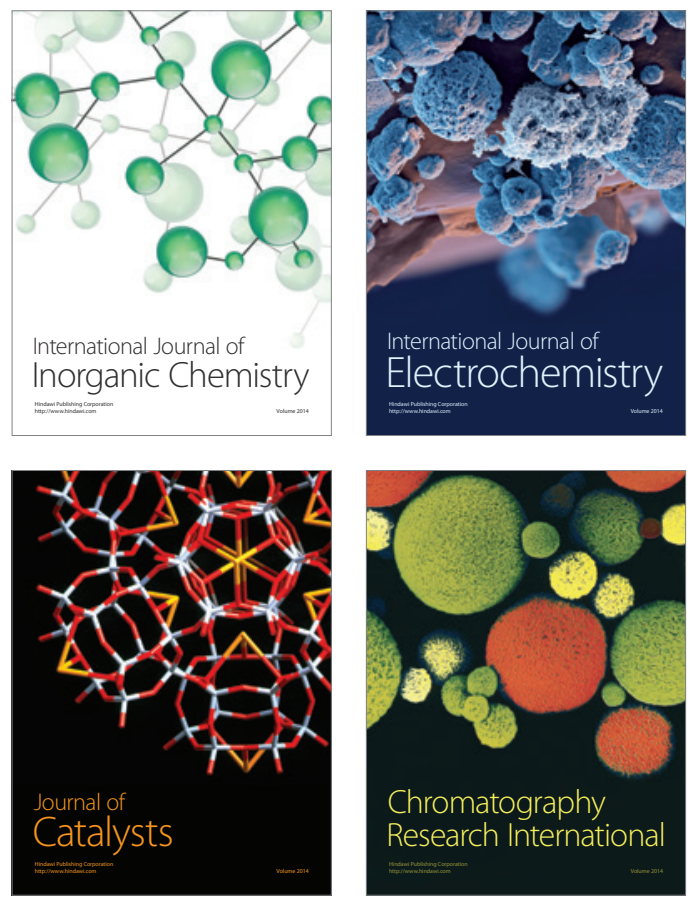
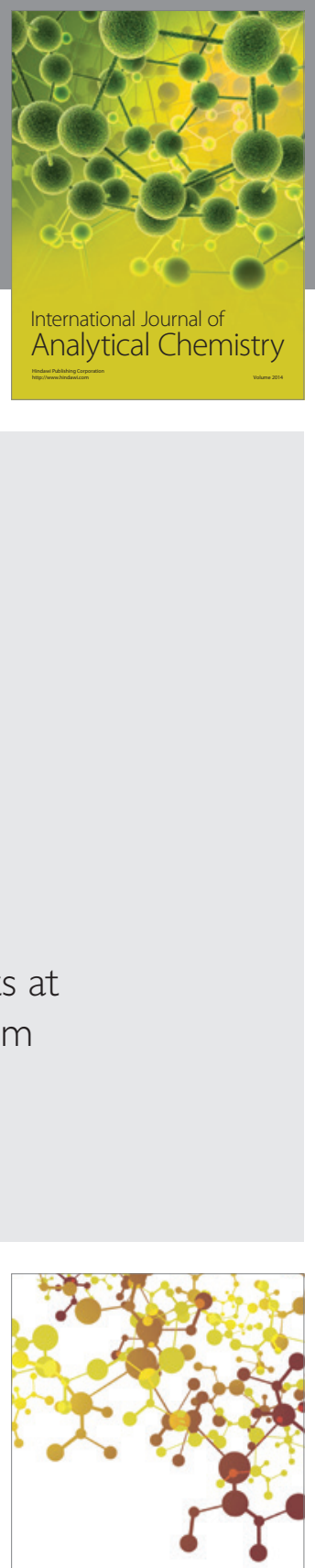

Journal of

Applied Chemistry
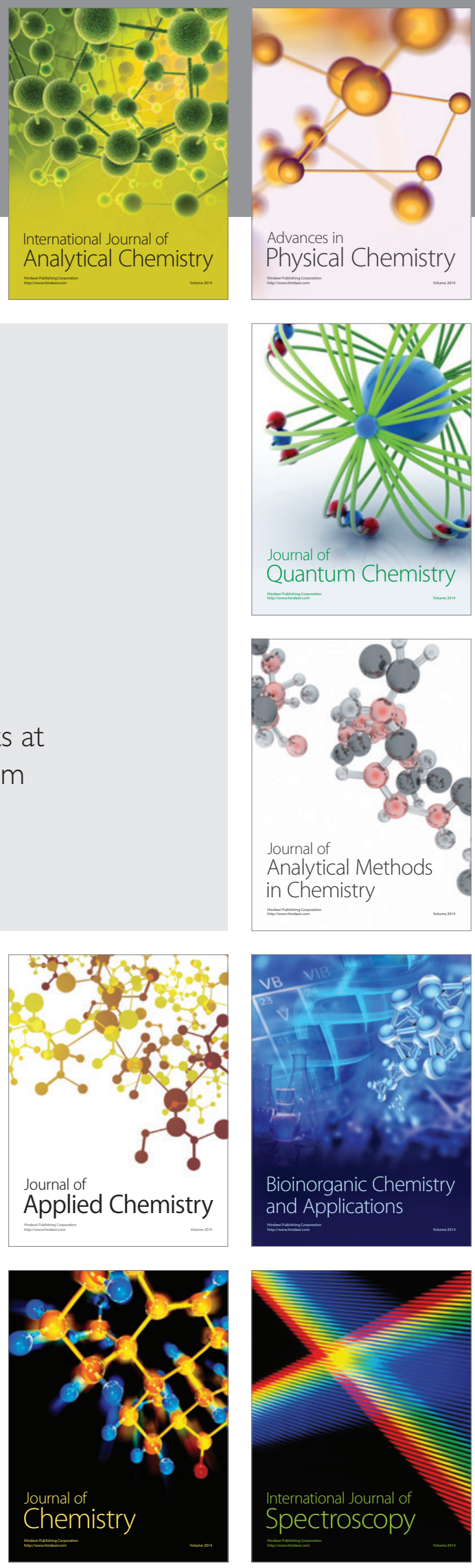